\title{
Variation of the argument of the Riemann $\xi$ function on vertical lines
}

by

\section{XiAnNAN Li (Stanford, CA)}

1. Introduction. Let $F(s)=\pi^{-s / 2} \Gamma(s / 2) \zeta(s)$, where as usual, $s=$ $\sigma+i t$ is a complex variable, and $\zeta(s)$ is the Riemann zeta function. Let $\xi(s)=\frac{s(s-1)}{2} F(s)$. Then $F(s)$ and $\xi(s)$ are real on the $\Re s=1 / 2$ line. We are interested in the zeros of the real or imaginary parts of $F(s)$ or $\xi(s)$ off the $1 / 2$ line. As will become clear in the proofs, there is essentially no difference in the behaviour of $F(s)$ or $\xi(s)$ in this situation.

Let $n_{\lambda}(T)$ denote the number of zeros of $\Re F(1 / 2+\lambda+i t)$ with $0<t \leq T$. For convenience, we can also assume that $\lambda>0$ since $F(s)=F(1-s)$. Let $N_{\lambda}(T)$ be the number of zeros of the zeta function in the region $\Re s \geq 1 / 2+\lambda$ and $0<\Im s \leq T$. Ki [2] improved a result of Levinson [4] and showed that

$$
n_{\lambda}(T)=\frac{T}{2 \pi} \log \frac{T}{2 \pi}-\frac{T}{2 \pi}+O\left(\left(N_{\lambda}(T)+1\right) \log T\right) .
$$

By an improvement of a result of Selberg by Jutila (see for instance 9.29 of [5]), we have $N_{\lambda}(T) \ll T^{1-a \lambda} \log T$ where $a$ is any constant less than 1 , so that the formula above is indeed an asymptotic formula. In the course of the proof, Ki also noted that the number of zeros of $\Re F(1 / 2+\lambda+i t)$ in the interval $[T, T+1]$ is $\ll \log T$. Similar results hold for the zeros of $\Im F(1 / 2+\lambda+i t)$.

The similarities between the results above and basic results on zeros of the zeta function are striking. However, in [3], Lagarias proves assuming $\mathrm{RH}$ for $0<\lambda<1 / 2$ and unconditionally for $\lambda \geq 1 / 2$ that the behaviour of these zeros is quite different from that of the zeros of the zeta function. Actually, Lagarias proved his results for slightly different functions, in essence for $R(t)=\Re \xi(1 / 2+\lambda+i t)$ and $I(t)=\Im \xi(1 / 2+\lambda+i t)$. He proved, unconditionally for $\lambda>1 / 2$ and conditionally assuming $\mathrm{RH}$ for $\lambda<1 / 2$, that the distributions of the spacings between normalized zeros of $R(t)$ or $I(t)$

2000 Mathematics Subject Classification: Primary 11M06; Secondary 11M26.

Key words and phrases: argument of $\zeta$, differenced L-functions, zero spacing. 
up to height $T$ converge to a limiting distribution with equal spacings of length 1 . This is in sharp contrast to the conjectured GUE distribution of zeros of $F(s)$ at $\Re s=1 / 2$. Lagarias also showed that the zeros of $I(t)$ and $R(t)$ interlace and are simple. That is, for any two consecutive zeros of $R(t)$, $\gamma_{1}$ and $\gamma_{2}$, there exists exactly one zero of $I(t)$ in $\left(\gamma_{1}, \gamma_{2}\right)$ and vice versa.

Lagarias's perspective was also somewhat different from that of Levinson in that instead of dealing with the real part or imaginary part of certain functions, he instead viewed his result in terms of differences of L-functions. Specifically he examined the entire functions $A(s)=\frac{1}{2}(\xi(s+\lambda)+\xi(s-\lambda))$ and $B(s)=\frac{1}{2 i}(\xi(s+\lambda)-\xi(s-\lambda))$, which can be considered as a family of entire functions depending on $\lambda$. By the functional equation and the fact that $\xi(\bar{s})=\overline{\xi(s)}$,

$$
\begin{aligned}
A(1 / 2+i t) & =\frac{1}{2}(\xi(1 / 2+i t+\lambda)+\xi(1 / 2+i t-\lambda)) \\
& =\frac{1}{2}(\xi(1 / 2+i t+\lambda)+\xi(1 / 2-i t+\lambda))=R(t) .
\end{aligned}
$$

Similarly, $B(1 / 2+i t)=I(t)$. Thus the correspondence between these two viewpoints is that the functions agree on the critical line.

Our results are unconditional analogues of the results in [3], which depended on $\mathrm{RH}$ in certain ranges. We will prove our results for $A(s)$ and $B(s)$ above, noting that our proofs apply with trivial changes to $\Re F(1 / 2+\lambda+i t)$ and $\Im F(1 / 2+\lambda+i t)$ as well. Our method also provides alternative routes to the results in [3], which we indicate in a later remark. Our first result is

THEOREM 1. Let

$$
A(s)=\frac{1}{2}(\xi(s+\lambda)+\xi(s-\lambda)) \quad \text { and } \quad B(s)=\frac{1}{2 i}(\xi(s+\lambda)-\xi(s-\lambda)) .
$$

Then for $\lambda>0$, the following holds unconditionally:

(i) $A(s)$ and $B(s)$ have almost all of their zeros on the line $\Re s=1 / 2$, with an exceptional set of zeros up to height $T$ not on that line of cardinality $\ll T^{1-a \lambda} \log ^{2} T$ for any $a<1$.

(ii) Let $0 \leq \gamma_{1} \leq \cdots \leq \gamma_{N}$ be the consecutive imaginary parts of the zeros of $A(s)$ or $B(s)$ and let $\tilde{\gamma}_{i}=2 \pi \gamma_{i} / \log \left(\gamma_{i}+1\right)$ be the normalized zeros. Similarly define normalized zeros for those zeros with negative imaginary part. The distribution of the spacings of the normalized zeros of $A(s)$ and $B(s)$ on the critical line, up to height $T$, converges to a limiting distribution with equal spacings of length 1 .

The first part of the theorem is essentially a result of Ki [2], and we will sketch a proof of it in the last section. The second part follows easily from the next theorem, which is our main focus.

Theorem 2. Let $R(t)=\Re \xi(1 / 2+\lambda+i t)$ and $I(t)=\Im \xi(1 / 2+\lambda+i t)$. For any $B>0$, there exists $E \subset[T, 2 T]$ with measure $\ll T / \log ^{B} T$ and 
containing $\ll T / \log ^{B} T$ zeros of $I(t)$ and $R(t)$ such that outside of this set, the zeros of $I(t)$ and $R(t)$ are simple, interlace and are regularly spaced.

The result above stated for $t \in[T, 2 T]$ implies the result for $t \in[0, T]$, by dyadic summation. This result will be proven in the next section. Throughout this paper, we shall assume a result that was essentially proven by Ki [2], namely that the number of zeros in $[T, T+1]$ of $R(t)$ or $I(t)$ is $\ll \log T$. Ki proved this for the imaginary and real parts of $F(s)$, and his proof, which is a standard application of the argument principle, applies without change to our case.

The result above is not the strongest possible. The exceptional set as stated is of size $T$ over any large power of $\log T$, but the power of $\log T$ can be replaced by a small power of $T$, depending on $\lambda$. Note that we expect it to be very difficult to prove the above result with no exceptional set at all, since that would imply RH. Our results extend in general to Dirichlet L-functions.

2. Proof of Theorem 2. For the rest of this note, let $\sigma=1 / 2+\lambda$ for $\lambda>0$, and $s=\sigma+i t$ for $t \in[T, 2 T]$. We start by noting that $R(t)$ has a zero precisely when $\arg \xi(s) \equiv \pi / 2 \bmod \pi$. Similarly $I(t)$ has a zero when $\arg \xi(s) \equiv 0 \bmod \pi$. Now by Stirling's formula,

$$
\begin{aligned}
& \log \left(\frac{s(s-1)}{2} \pi^{-s / 2} \Gamma\left(\frac{s}{2}\right)\right) \\
& \quad=-\frac{s}{2} \log \pi+\left(\frac{s}{2}-\frac{1}{2}\right) \log \frac{s}{2}-\frac{s}{2}+\frac{\log 2 \pi}{2}+\log \frac{s(s-1)}{2}+O(1 / s),
\end{aligned}
$$

from which we get

$$
\arg \left(\frac{s(s-1)}{2} \pi^{-s / 2} \Gamma\left(\frac{s}{2}\right)\right)=\frac{t}{2} \log \frac{t}{2 \pi}-\frac{t}{2}+O(1) .
$$

To understand $\arg \xi(s)$, we argue that the above dominates $\arg \zeta(s)$ outside of some exceptional set, so we need to examine $\arg \zeta(s)$. Suppose that $t \in$ $[T, 2 T]$ for some large $T$. By Selberg, we have the standard identity (see e.g. $[5,14.20])$

$$
\begin{aligned}
\frac{\zeta^{\prime}}{\zeta}(s)= & -\sum_{n<x^{2}} \frac{\Lambda_{x}(n)}{n^{s}}+\frac{x^{2(1-s)}-x^{1-s}}{(\log x)(1-s)^{2}}+\frac{1}{\log x} \sum_{q \geq 1} \frac{x^{-2 q-s}-x^{-2(2 q+s)}}{(2 q+s)^{2}} \\
& +\frac{1}{\log x} \sum_{\varrho} \frac{x^{\varrho-s}-x^{2(\varrho-s)}}{(s-\varrho)^{2}} \\
= & -\sum_{n<x^{2}} \frac{\Lambda_{x}(n)}{n^{s}}+\frac{1}{\log x} \sum_{\varrho} \frac{x^{\varrho-s}-x^{2(\varrho-s)}}{(s-\varrho)^{2}}+O\left(\frac{x}{T^{2}}\right),
\end{aligned}
$$


where

$$
\Lambda_{x}(n)= \begin{cases}\Lambda(n) & \text { for } 1 \leq n \leq x, \\ \Lambda(n) \frac{\log \left(x^{2} / n\right)}{\log x} & \text { for } x \leq n<x^{2} .\end{cases}
$$

The last sum is over nontrivial zeros of $\zeta$. This sum is only bounded nicely if we stay away from zeros of $\zeta$. Specifically, for $A$ a parameter to be chosen later, define $E_{1}=E_{1}(A)$ to be the union of all rectangles with vertices $\sigma_{0}+i \gamma-i \log ^{A} T, \sigma_{0}+i \gamma+i \log ^{A} T, 1+i \gamma+i \log ^{A} T$, and $1+i \gamma-i \log ^{A} T$, over all $\gamma \in[T, 2 T]$ such that $\zeta(\beta+i \gamma)=0$ for some $\beta \geq \sigma_{0}$. Then $\mu\left(E_{1}\right) \ll$ $N_{\lambda / 2}(T) \log ^{A} T \ll T / \log ^{B} T$, and $E_{1}$ contains $\ll T / \log ^{B} T$ zeros in total, for any $B>0$. Indeed, $E_{1}$ consists of $\ll N_{\lambda / 2}(T)$ intervals each of which contains $\ll \log ^{A+1} T$ zeros of $R(t)$ and $I(t)$, so $E_{1}$ contains $\ll T / \log ^{B} T$ zeros in total, for any $B>0$.

Let $\delta$ be a constant satisfying $0<\delta<1$; we will discuss choices for $\delta$ later. Set $x=\log ^{2(1+\delta) / \lambda} T$. Then we have the following lemma.

Lemma 1. There is a choice of $A$ depending only on $\delta$ and $\lambda$ such that outside of $E_{1}=E_{1}(A)$,

$$
\frac{\zeta^{\prime}}{\zeta}(s)=-\sum_{n<x^{2}} \frac{\Lambda_{x}(n)}{n^{s}}+O\left(\frac{1}{\log ^{\delta} T}\right) .
$$

Proof. Let $\sigma_{0}=1 / 2+\lambda / 2$. Outside of $E_{1}, \sum_{\varrho}\left(x^{\varrho-s}-x^{2(\varrho-s)}\right) /(s-\varrho)^{2}$ is small. As usual, write $\varrho=\beta+i \gamma$. We split the sum over $\beta \geq \sigma_{0}$ and $\beta<\sigma_{0}$. The sum over $\beta \geq \sigma_{0}$ is

$$
\ll \frac{x^{2(1-\sigma)}}{\log ^{A-1} T} .
$$

Here we bounded the numerator by $x^{2(1-\sigma)}$, noted that $|s-\varrho| \geq \log ^{A} T$ for $\beta \geq \sigma_{0}$ and $s \notin E_{1}$, and used the bound of $\log T$ on the number of zeros $\varrho$ of $\zeta$ satisfying $\Im \varrho \in[T, T+1]$.

Similarly, the sum over $\beta<\sigma_{0}$ is

$$
\ll x^{-\lambda / 2} \log T \ll \frac{1}{\log ^{\delta} T},
$$

by the definition of $x$. Since $x$ is a power of $\log T$, we may pick $A$ sufficiently large such that the first bound is $\ll \frac{1}{\log ^{\delta} T}$. Specifically, we set $A=(1-$ $2 \lambda) 2(1+\delta) / \lambda+1+\delta$. The result then follows.

To grasp the size of the sum over primes appearing in (1), we will refer to Corollary 9.5 in [1] which states

Lemma 2. Let $A(s)=\sum_{n \leq N} a(n) / n^{s}$ be a Dirichlet polynomial. Let $T \geq 1$ and let $s_{r}=\sigma+i t_{r}(r=1, \ldots, R)$ be points with $T<t_{1}<\cdots<t_{R}$ 
$<2 T$ and $t_{r+1}-t_{r} \geq 1$. Then

$$
\sum_{r=1}^{R}\left|A\left(s_{r}\right)\right|^{2 k} \ll\left(T+N^{k}\right) \log \left(2 N^{k}\right)\left(\sum_{n \leq N} d_{k}(n)|a(n)|^{2} n^{-2 \sigma}\right)^{k},
$$

where $d_{k}(m)$ denotes the number of factorizations of $m$ as a product of $k$ numbers, and the implied constant is absolute.

We apply this lemma to $A(s)=\sum_{n<x^{2}} \Lambda_{x}(n) / n^{s}$, so that $N=x^{2}=$ $\log ^{4(1+\delta) / \lambda} T \ll \log ^{8 / \lambda} T$. In our case, $\sigma=1 / 2+\lambda$ so $\sum_{n} d_{k}(n)|a(n)|^{2} n^{-2 \sigma}$ converges. Hence

$$
\sum_{r=1}^{R}\left|A\left(s_{r}\right)\right|^{2 k} \ll_{k, \lambda} T \log T
$$

for any $k$ satisfying $N^{k} \ll T$. Since $N$ is a power of $\log T$, we may pick $k$ to be as large a constant as we like. Now suppose that $A\left(s_{r}\right) \gg \log ^{1-\delta} T$ for each $1 \leq r \leq R$. Then $R \ll T / \log ^{B} T$ for any fixed $B>0$. Here, for a given $B$, the choice of $k$ will depend only on $B$ and $\delta$. Now let

$$
E_{2}=\left\{t \in[T, 2 T]:|A(\sigma+i t)|>\log ^{1-\delta} T\right\} .
$$

From the discussion above, $E_{2}$ has at most $\ll T / \log ^{B} T$ points that are separated from each other by at least 1 , and thus has $\ll T / \log ^{B-1} T$ zeros of $R(t)$ or $I(t)$. Clearly, the measure of $E_{2}$ is $\ll T / \log ^{B} T$.

Let $E=E_{1} \cup E_{2}$ be our exceptional set. Then in intervals outside of $E$,

$$
\frac{d}{d t} \arg \xi(\sigma+i t)=\frac{1}{2} \log \frac{t}{2 \pi}+O\left(\log ^{1-\delta} T\right),
$$

where the main term comes from logarithmic differentiation of the gamma factor and the error term from $\frac{\zeta^{\prime}}{\zeta}(s)$. Specifically, the main term arises from

$$
\begin{aligned}
\Im \frac{d s}{d t} \frac{d}{d s}\left(\log \left(\pi^{-s / 2}\right)+\log \Gamma(s / 2)\right. & +s(s-1) / 2) \\
& =\Im i\left(-\frac{1}{2} \log \pi+\frac{1}{2} \log \frac{s}{2}+O(1 / s)\right) \\
& =\frac{1}{2} \log \frac{t}{2}+O(1) .
\end{aligned}
$$

Thus, the argument is a strictly increasing function of $t$ for large $T$ and so the zeros of $R(t)$ and $I(t)$ are simple and strictly interlace.

It remains to examine the normalized spacings. By integrating (2), we find that for any $\Delta \ll 1 / \log T$,

$$
\arg \xi(s+i \Delta)-\arg \xi(s)=\frac{\Delta}{2} \log \frac{t}{2 \pi}+O\left(\log ^{-\delta} T\right) .
$$


In particular, if $\gamma_{1}<\gamma_{2}$ were consecutive zeros of $R(t)$ in $[T, 2 T]$, we claim that

$$
\gamma_{2}-\gamma_{1}=\frac{2 \pi}{\log \frac{\gamma_{1}}{2 \pi}}+O\left(\frac{1}{\log ^{1+\delta} T}\right) .
$$

Indeed, by the monotonicity of $\arg \xi(\sigma+i t)$,

$$
\arg \xi\left(\sigma+i \gamma_{2}\right)-\arg \xi\left(\sigma+i \gamma_{1}\right)=\pi .
$$

The claim then follows from (3). Consequently, the normalized spacing is $1+O\left(1 / \log ^{\delta} T\right)$. Let $\psi$ be the unique zero of $I(t)$ in $\left(\gamma_{1}, \gamma_{2}\right)$. Then similarly

$$
\psi-\gamma_{1}=\frac{\pi}{\log \frac{\gamma_{1}}{2 \pi}}+O\left(\frac{1}{\log ^{1+\delta} T}\right)
$$

so that $\psi$ is actually in the middle of $\left(\gamma_{1}, \gamma_{2}\right)$. Of course, the same holds with the roles of $R(t)$ and $I(t)$ reversed. Note that the results above all hold for any $\delta \in(0,1)$.

REMARK. Our method of proof also provides a direct alternative to the proof of results in [3]. Indeed, if we assume RH, then Titchmarsh [5, 14.5.1] gives

$$
\frac{\zeta^{\prime}}{\zeta}(s) \ll \log ^{1-2 \lambda} T \quad \text { for } 0<\lambda<1 / 2,
$$

so if we take $\delta=2 \lambda$, we would have no exceptional set. For $\lambda>1 / 2$, things are even simpler as we have unconditionally $\frac{\zeta^{\prime}}{\zeta}(s) \ll 1$. Finally, for $\lambda=1 / 2$, an unconditional result is still possible by using the fact that

$$
\frac{\zeta^{\prime}}{\zeta}(s) \ll \frac{\log T}{\log \log T} \quad \text { for } T \geq 2,
$$

from $[5,5.17 .4]$. Here the error terms with $1 / \log ^{\delta} T$ would be replaced with $1 / \log \log T$.

3. An analogue of Ki's result. Here we will provide a sketch of the proof of Theorem 1(i), leaving out those details which are readily available in $[2]$. Let

$$
G(z):=\xi(1 / 2+i z)
$$

and

$$
H(z):=G(z-i \lambda)+G(z+i \lambda), \quad J(z):=G(z-i \lambda)-G(z+i \lambda) .
$$

By the definition of $G$ and by the functional equation for $\xi$, the two functions above correspond to the real and imaginary parts of $\xi(1 / 2+\lambda+i t)$ when $z=t$ is real.

The main idea of the proof is to show that there is some small exceptional set, outside which $H(z)$ and $J(z)$ have only real zeros, and so outside of a 
small exceptional set, $A(s)$ and $B(s)$ only have zeros on the $1 / 2$ line. The key to showing this will be to show that $G(z-i \lambda)$ and $G(z+i \lambda)$ have different sizes when $z$ is not real. The exceptional set will essentially consist of neighbourhoods around the zeros of $\zeta(s)$ for $\Re s \geq 1 / 2+\lambda$. To be precise, let $\left\{s_{i}\right\}_{i=1}^{\infty}$ denote those zeros of $\zeta$ with real part $\geq 1 / 2+\lambda$ and $\Im s_{i} \leq \Im s_{i+1}$ for all $i$. Let

$$
\mathcal{R}(\kappa)=\left\{z:|\Re z| \geq\left|\Im s_{1}\right| \text { and }\left|\Re z-\Im s_{k}^{*}\right|>\kappa, s_{k}^{*}=s_{k} \text { or } \bar{s}_{k} \forall k\right\} .
$$

The exceptional set will be the complement of $\mathcal{R}(\kappa)$. Our unconditional analogue of Theorem B of [2] is

LEMma 3. There is some $\kappa>0$ such that all the zeros $z$ with $|z|>\kappa$ of $H(z)$ or $J(z)$ in $\mathcal{R}(\kappa)$ are real.

From now on, we will restrict the discussion to $H(z)$, the situation with $J(z)$ being the same. The points which are not in $\mathcal{R}(\kappa)$ are all within a constant distance of a zero of $\zeta(s)$ with $\Re s \geq 1 / 2+\lambda$. The number of such zeros is $\ll T^{1-a \lambda} \log T$ for any $a<1$. The number of zeros of $H(z)$ for $T \leq \Re z \leq T+C$ is $\ll \log T$ for any constant $C$. As we mentioned in the introduction, the proof of this proceeds along standard lines using the argument principle, and we refer the reader to the proof of Proposition 2.2 of [2] for more details. Thus, the number of zeros of $H(z)$ outside of $\mathcal{R}(\kappa)$ is $\ll T^{1-a \lambda} \log ^{2} T$, uniformly in $\lambda$.

The proof of Lemma 3 is the same as the proof of Theorem B in [2]. We provide a sketch below, referring the reader to details which are available in [2] whenever possible.

Proof of Lemma 3. Set $z=x-i y \in \mathcal{R}(\kappa)$ with $x>1$ and $y>0$. By equations (3.6) and (3.7) in [2], for some constants $H_{0}, H_{1}, \sigma_{1}>0$, explicitly described in [2],

$$
\begin{aligned}
\log \left|\frac{G(\bar{z}-i \lambda)}{G(z-i \lambda)}\right| & \leq 4 y\left(H_{0} \frac{\log (\kappa+x)}{\kappa^{2}}+H_{0} \sum_{n \geq 1} \frac{\log x}{(\kappa+n)^{2}}-\frac{\lambda H_{1} \log x}{1+\sigma_{1}^{2}}\right) \\
& =4 y(\log x)\left(H_{0}\left(\frac{1}{\kappa}+\frac{1}{\kappa^{2}}\right)-\frac{\lambda H_{1}}{1+\sigma_{1}^{2}}\right)(1+o(1)) \\
& =: 4 y(\log x) \delta(1+o(1))
\end{aligned}
$$

where we have set

$$
\delta=H_{0}\left(1 / \kappa+1 / \kappa^{2}\right)-\lambda H_{1} /\left(1+\sigma_{1}^{2}\right) .
$$

We then pick $\kappa$ such that $\delta>0$ and so

$$
\left|\frac{G(\bar{z}-i \lambda)}{G(z-i \lambda)}\right| \leq e^{-4 y \log x \delta} .
$$


Now we have that

$$
\begin{aligned}
|H(z)| & =G(z-i \lambda)+G(z+i \lambda) \\
& \geq|G(z-i \lambda)|\left(1-\frac{G(z+i \lambda)}{G(z-i \lambda)}\right) \\
& =|G(z-i \lambda)|\left(1-\frac{G(\bar{z}-i \lambda)}{G(z-i \lambda)}\right)>0
\end{aligned}
$$

for $z \in \mathcal{R}(\kappa)$, and for $|z| \geq \kappa$. Thus $H(z) \neq 0$ for $z \in \mathcal{R}(\kappa)$ with $|z| \geq \kappa$ and $y>0$. Since $H(z)=\overline{H(\bar{z})}$ and $H(-z)=H(z)$, the same result holds true for $y<0$, from which we deduce that all zeros of $H(z)$ with $z \in \mathcal{R}(\kappa)$ and $|z| \geq \kappa$ must be real.

Acknowledgements. I am grateful to Professor Soundararajan for his guidance throughout the making of this paper. I would like to thank Bob Hough, Vorrapan Chandee and Leo Goldmakher for editorial comments. I am also indebted to the referee for helpful suggestions.

\section{References}

[1] H. Iwaniec and E. Kowalski, Analytic Number Theory, Colloq. Publ. 53, Amer. Math. Soc., Providence, RI, 2004.

[2] H. Ki, On a theorem of Levinson, J. Number Theory 107 (2004), 187-197.

[3] J. C. Lagarias, Zero spacing distributions for differenced L-functions, Acta Arith. 120 (2005), 159-184.

[4] N. Levinson, On theorems of Berlowitz and Berndt, J. Number Theory 3 (1971), 502-504.

[5] E. C. Titchmarsh, The Theory of the Riemann Zeta-Function, 2nd ed., Oxford Univ. Press, New York, 1986.

Department of Mathematics

Stanford University

Stanford, CA 94305, U.S.A.

E-mail: xli@math.stanford.edu

Received on 22.5.2008

and in revised form on 19.10.2008 